\title{
Construcción de paz y convivencia escolar a través de la investigación como estrategia pedagógica ${ }^{1}$
}

\section{Building peace and school coexistence through Research as a Pedagogical Strategy}

DOI: http://dx.doi.org/10.17981/cultedusoc.9.1.2018.06

Fecha de recepción: 03/04/2018. Fecha de aceptación: 03/07/2018

Yudy Cardona-Jaramillo ${ }^{2}$ (D); Luzmila Patricia Bado-Herrera ${ }^{3}$

Wilson Pedraza-Álvarez; Lucelis del pilar Bado-Herrera; Celene Margarita-Castañeda; Edgar Caballero-Jiménez; Rosa Isabel Castillo-Bujato; Alba Cristina Fuentes-Barraza; Alberto Manuel Mendinueta-Roca; Maryuris María Montaño-Fernández; Elsy Orellano-Castillo; Farides Ester Pérez-Araque; Sebastiana María Sarmiento-Carrillo ${ }^{4}$

Para citar este artículo

Cardona-Jaramillo, Y., Bado-Herrera, L., Pedraza-Álvarez, W., Bado-Herrera, L., Margarita-Castañeda, C., Caballero-Jiménez, E., Castillo-Bujato, R., Fuentes-Barraza, A., Mendinueta-Roca, A., Montaño-Fernández, M., Orellano-Castillo. E., Pérez-Araque, F. y Sarmiento-Carrillo, S. (2018). Construcción de paz y convivencia escolar a través de la investigación como estrategia pedagógica. Cultura. Educación y Sociedad 9(1), 83-94. DOI: http://dx.doi.org/10.17981/cultedusoc.9.1.2018.06

\section{Resumen}

El conflicto es inherente a las relaciones humanas, es así, como la dinámica se reproduce en cualquier ámbito donde interactúen personas. Sin embargo, la violencia es sólo una de las posibilidades para resolver una situación conflictiva, en este artículo se mostrarán diversas maneras de solucionar los problemas desde la pedagogía. El propósito de la investigación fue generar espacios de construcción de paz y convivencia a través de la investigación como estrategia pedagógica (IEP). El Estudio se centró bajo el enfoque cualitativo, desde el tipo de investigación acción. La población de este estudio estuvo constituida por cuarenta (40) estudiantes del grado sexto de bachillerato de la IED Humberto Velázquez García. Los resultados evidenciaron poca comprensión y respeto de los derechos humanos, falta de concepción democrática, lo que desmejora la construcción de relaciones interpersonales e institucionales y culturales justas, propiciando falencias en el fortalecimiento de los valores en las instituciones.

Palabras clave: construcción de paz, convivencia escolar, investigación como estrategia pedagógica.

\begin{abstract}
The conflict is inherent in human relations, is thus, as Dynamics is reproduced in any area where people interact. However, violence is only one of the possibilities to resolve a conflict situation, this article will show different ways of solving problems from the pedagogy. The purpose of the investigation was to generate spaces for building peace and coexistence through research as a pedagogical strategy (IEP). The study focused under the qualitative approach, since the type of action research. This study population consisted of forty (40) students of the sixth grade of Bachelor of the FDI Humberto Velázquez Garcia. The results showed little understanding and respect of human rights, lack of democratic conception, what fair demotion the construction of interpersonal and institutional and cultural relations, leading to flaws in the strengthening of the values in the institutions.
\end{abstract}

Keywords: building peace, co-existence in school and research as a pedagogical strategy.

\footnotetext{
${ }^{1}$ Este artículo ha sido derivado del Programa de Fortalecimiento de la Cultura Ciudadana y Democrática CT+I a través de la IEP apoyada en TIC en el Departamento de Magdalena: CICLON

${ }^{2}$ Psicóloga especialista en Psicoterapia Infantil de la Universidad de la Costa, CUC. Correo de correspondencia: yudycardonaj@ gmail.com

${ }^{3}$ Docente de la Institución Educativa Departamental Humberto Velázquez García. Líder del grupo de investigación Sembradores de Valores. Correo de correspondencia: luzmilabado@hotmail.com.

${ }^{4}$ Docentes de la Institución Educativa Departamental Humberto Velázquez García. Grupo institucional.
} 


\section{Introducción}

La educación es un proceso dinámico que permite la evolución del hombre en todas sus dimensiones; social, cultural, ética y escolar. Busca formar seres integrales mediante la formulación de bases sólidas que marquen un proceso en el desarrollo continuo, estructurando valores y actitudes presentes dentro de la sociedad. En el campo educativo se han desarrollado acciones de formación importantes para propiciar cambios de paradigmas mentales y conductuales, involucrando familia, escuela y sociedad, a partir de los hábitos, creencias, actitudes que incluyen los valores.

Partiendo de las transformaciones curriculares practicadas en el contexto educativo, se evidencia que desarrollar dentro del aula trabajo cooperativo es una técnica eficaz, permitiendo al alumno obtener una participación activa, adquirir respeto hacia las ideas de los demás, reconociendo que en equipo se pueden resolver problemas, aprendiendo así de las experiencias compartidas. Por tal razón la integración de la IEP y las TIC para la construcción de paz y convivencia en el aula de clase se convierte en una estrategia pedagógica, generando espacios de reflexión y discusión que propicien una formación en valores y competencias ciudadanas.

La educación desde las diversas áreas del saber busca generar una integración ética, orientada en adquirir competencias cognitivas de acuerdo a los estándares del Ministerio de Educación y a las competencias sociales de convivencia, cultura ciudadana y civismo. Para descubrir de esta manera clara la forma en cómo los individuos asumen retos, barreras y logros alcanzados en las relaciones humanas. Por tanto, el clima educativo propende por la participación activa de toda la comunidad educativa, los cuales unen esfuerzos por tener una educación con calidad, en la cual los hábitos de convivencia promuevan el respeto, inclusión y dignidad social de la escuela.

El país a nivel educativo a promovido arduamente la construcción de una cultura de paz, con legislaciones como la Ley 1732 de 2014 y su decreto reglamentario 1038 de 2015, en los que establece la cátedra de la paz en todas las instituciones educativas del país, con el objetivo de "crear y consolidar un espacio para el aprendizaje, la reflexión y el diálogo sobre la cultura de la paz y el desarrollo sostenible que contribuya al bienestar general y el mejoramiento de la calidad de vida de la población".

La IED Humberto Velázquez García ha sido creada con el ánimo de ser una institución de alta calidad con educación inclusiva para el corregimiento de Sevilla y Palomar ubicado en Zona Bananera-Magdalena, poblándose masivamente en las últimas décadas, sin embargo, se vienen presentando problemas de violencia generalizada, marginación y pobreza, atentado contra los derechos de la comunidad educativa, y que hacen eco en sus hogares.

Toda esta situación se ha presentado a causa de procesos dinámicos del país inconscientemente, quiebres y altibajos que revisten retos y estrategias de acción paralelas para la construcción de paz y convivencia, trayendo como consecuencias, que los espacios o aulas de clases entre los estudiantes, padres de familia, comunidad académica se observen fallas en los procesos de negociación, paz, intolerancia, disminución en valores como principios institucionales, y equidad en espacios colaborativos de aprendizajes. 
De seguir con estas situaciones, podría aumentar la falta de valores, actitudes, comportamientos y estilos de vida que rechazan la violencia y previenen los conflictos atacando, mediante el diálogo y negociación entre los estudiantes y comunidad educativa. Por lo anterior el grupo de investigación se organizó para buscar soluciones desde la pedagogía a la problemática, respecto a la violencia escolar que se presenta crecidamente en el grado sexto de la institución. Debido a las situaciones conflictivas observadas por los docentes dentro del aula e instalaciones escolar, surgió la necesidad de organizar y ejecutar pautas, con el objetivo de generar espacios de construcción de paz y convivencia a través de la investigación como estrategia pedagógica (IEP).

\section{Elementos conceptuales de}

la construcción de paz y

convivencia escolar a través

de la IEP apoyada en TIC

El sistema educativo es una institución social que surge para cumplir una serie de funciones encaminadas a la formación de estudiantes, generando así, espacios de socialización entre los individuos, con la finalidad formar a hombres y mujeres que las sociedades necesitan para funcionar, desarrollando rasgos de personalidad, valores, actitudes y comportamientos básicos requeridos para la interacción social. Actualmente se ha enfatizado sobre la importancia de la institución escolar como agente de socialización al favorecer el fortalecimiento de los valores y actitudes dominantes de las colectividades, sin embargo, el sistema educativo es una realidad compleja puesto que está inmerso dentro de la sociedad, por ello, está estrechamente vinculado a los ámbitos políticos, económicos y sociales. (Fernández, 2006).

Según Samper \& Maussa. (2014). Colombia, ha sido un país que ha contado con más de cincuenta años de conflicto armado interno, no obstante, el Ministerio de Educación actualmente promueve la educación para la participación democrática como mecanismo para superar las dificultades, orientados hacia la construcción de una cultura de paz. Por lo cual la escuela se convierte en la institución adecuada para brindar la formación pertinente para la adquisición de estándares de competencias ciudadanas, reconociendo los derechos fundamentales; lo cual ayudara a generar la convivencia pacífica, con el reconocimiento de las diferencias, desde esta perspectiva la escuela sería un espacio de participación ciudadana que promueva la equidad, la inclusión y la reconciliación.

Por su parte, Robles, (2015). Plantea que, la violencia más allá de un problema o una situación particular aislada, puesto que esta se presenta en la cotidianidad de las escuelas, además, estas situaciones se legitiman, justifican, producen y reproducen, sus funcionalidades en diversos contextos, donde las dinámicas y efectos en los individuos generan conductas y relaciones discriminatorias en los individuos de las instituciones educativas.

Así mismo, Fernández (2006) asegura que para comprender la naturaleza de la escuela es necesario partir de un análisis crítico de estructuras sociales, como también de los intereses y conflictos que le conforman. Entendiendo que históricamente, la escuela ha sido un lugar donde se han presentado conflictos, pero esta también se ha preocupado por buscar y construir alternativas de 
solución. Por ello, la educación debe ser analizada desde una perspectiva diferente, asumiendo que ésta contribuye en formación de ciudadanos capaces de modificar relaciones sociales existentes, donde la paz debe considerarse como una opción alcanzable en espacios escolares.

La construcción de paz en la escuela no se concibe para evitar que se sigan generando conflictos, sino para permitir el abordaje de sus raíces estructurales, interviniendo a partir de la democracia y la justicia, construyendo espacios equitativos de participación estudiantil, donde se asegure que todos los sujetos tengan los medios para participar en el desarrollo de su propia sociedad, en ese sentido la convivencia debe estar planteada hacia una concepción democrática, propiciando la construcción de relaciones interpersonales, institucionales y culturales justas que ofrezcan a todos los estudiantes un acceso equitativo a la educación de calidad, (Carbajal, 2013).

Es así como surge, en la época moderna, la preocupación por una protección integral a los estudiantes, observándolos como sujetos potenciales en nuevas acciones de política legislativa en casi todos los países del mundo, lo que ha generado la consagración de los derechos, a través de preceptos, valores y principios constitucionales, generando un análisis científico. (Herrera, 2013).

Por su parte, el termino convivencia hace alusión a la relación que se da entre individuos que conviven o que ocupan un mismo espacio de socialización, este término se utiliza particularmente para hablar del hecho de vivir en armonía, tolerando y respetando las diferencias en un ambiente pacifico, donde se respetan las normas y valores que regulan el trato mutuo, (Pick, 2002). Sin embargo, la convivencia escolar es un término que ha sido empleado en el ámbito educativo para disminuir los índices de violencia en la escuela, donde se han implementado prácticas autoritarias que reprimen y castigan a los estudiantes, generando violencia estructural, donde el docente $o$ directivos mantienen una relación vertical, adjudicando al estudiante un rol pasivo como en la educación tradicional, (Carbajal, 2013).

Ante la cita en mención, esa concepción de convivencia escolar no permite la construcción de paz puesto que genera espacios desiguales donde el estudiante no tiene derechos a la participación, ni al desarrollo de pensamiento crítico que permita fomentar la capacidad de solucionar conflictos, por tal razón se plantea la integración con la construcción de paz, para fomentar escenarios democráticos de participación estudiantil.

Para integrar la construcción de paz y convivencia escolar en las instituciones educativas es necesario incorporar prácticas equitativas, igualitarias $\mathrm{y}$ participativas que contribuyan a construir y sustentar una cultura de paz positiva en el salón de clases, esto es posible mediante prácticas pedagógicas apoyadas en las tecnologías de la información y comunicación que estimulen la participación, pensamiento crítico y el empoderamiento de los estudiantes, por lo cual se plantea la inclusión de la investigación como estrategia pedagógica como estrategia para fomentar la construcción de paz y la convivencia escolar, considerando los indicadores de los elementos conceptuales de la construcción de paz y convivencia escolar de acuerdo al Ministerio de Educación (2016), Cerdas (2015), Carbajal (2013) y Fernández (2006), (ver tabla 1). 
Tabla 1

Indicadores de los elementos conceptuales de la construcción de paz y convivencia escolar

\begin{tabular}{|c|c|}
\hline Teóricos & $\begin{array}{l}\text { Indicadores de los elementos conceptuales } \\
\text { de la construcción de paz y convivencia escolar }\end{array}$ \\
\hline \multirow[t]{2}{*}{$\begin{array}{l}\text { Ministerio de } \\
\text { Educación (2016). }\end{array}$} & $\begin{array}{l}\text { Ejercicio activo de los derechos humanos en el contexto escolar, familiar y } \\
\text { comunitario. }\end{array}$ \\
\hline & Reconocimiento, comprensión y respeto de los derechos humanos. \\
\hline Cerdas (2015). & $\begin{array}{l}\text { Interiorización individual y colectiva de relaciones pacíficas y armoniosas entre los } \\
\text { seres humanos. }\end{array}$ \\
\hline \multirow[t]{2}{*}{ Carbajal (2013). } & $\begin{array}{l}\text { Concepción democrática, construcción de relaciones interpersonales, institucionales } \\
\text { y culturales justas, y acceso equitativo a la educación de calidad. }\end{array}$ \\
\hline & Disminuir los índices de violencia en la escuela. \\
\hline Fernández (2006). & Fortalecimiento de los valores y actitudes dominantes de las colectividades. \\
\hline
\end{tabular}

Fuente: elaboración propia (2018).

Por otro lado, Mejía (2011), expresa que la investigación como estrategia pedagógica (IEP), es una metodología encaminada a fomentar el interés en la población estudiantil por la investigación científica y tecnológica, en donde el maestro acompaña a sus educandos en la búsqueda del saber a través de una acción dialógica que incluye el texto, la disciplina y el método científico. El mismo autor señala, que esta herramienta permite llevar la investigación a la esfera educativa y pedagógica, que además genera una reelaboración del campo de éstas, así como de los mecanismos prácticos y de acción que las han constituido.

Actualmente la sociedad del conocimiento ha transformado el mundo educativo, donde la ciencia, la tecnología y la innovación inciden en diferentes de forma significativa en la vida de los estudiantes, hecho que ha producido un cambio en los pensamientos $\mathrm{y}$ acciones de los individuos frente al proceso de enseñanza. (Cárdenas \& Angulo, 2016)

La IEP implica el proceso de sistematización de maestros(as) el cual apun- ta a describir y comprender lo sucedido durante las experiencias pedagógicas, con el fin de explicar los resultados de tales experiencias y las razones por las que se obtuvieron. De esta manera, los maestros estarán en capacidad de hacer reflexiones a los procesos a partir de los roles que cada uno cumple, e identificar estrategias para mejorar las experiencias futuras (Mejía, 2011).

En la actualidad las nuevas tecnologías de la información y comunicación (TIC) han tomado un gran auge y su integración al contexto educativo ha generado grandes cambios en el proceso de enseñanza-aprendizaje, estas giran en torno a tres medios básicos: la informática, la microelectrónica y telecomunicaciones; de manera interactiva e interconectadas, lo que permite conseguir nuevas realidades comunicativas, impactando de manera positiva la escuela (Belloch, 2012).

Las TIC son tecnologías para almacenar, recuperar, procesar y comunicar la información. Existe una variedad de recursos electrónicos que se encuadran dentro del concepto de TIC como; televisión, teléfonos, videos, ordenadores, en- 
tre otros. Sin embargo, los medios más representativos de la sociedad actual son los ordenadores que nos permiten utilizar diferentes aplicaciones informáticas (presentaciones, aplicaciones multimedia, programas ofimáticos y redes de comunicación (Belloch, 2012).

Se hace necesario que estos elementos conceptuales de la construcción de paz y convivencia escolar a través de la IEP apoyada en TIC, sean comunicados con los participantes, personal académicos y comunidad, esta triangulación posibilitará la creación de estrategias institucionales, con elementos comunes mediante el conocimiento y la defensa de los derechos humanos, la mediación, la necesidad de reparación del daño, la reconciliación y el perdón.

Para Ramírez y Hugueth (2017), la acción de la comunicación para la efectividad de gestión en las organizaciones, ha experimentado la inclusión de esta variable en las políticas o filosofía del proceso de cambio institucional, siendo esta una herramienta hoy día para integrar su público la cultura corporativa $\mathrm{y}$ armonizar las líneas de mando a seguir, minimizando los conflictos que se pudieran presentar entre estos, considerando la retroalimentación, un indicador de éxito en los espacios de trabajo donde se genere.

Es probable que la comunicación deficiente sea la fuente de los conflictos interpersonales que se citan con frecuencia dentro de las instituciones, debido a que los individuos se comunican cerca de 70 por ciento de las horas que están en vigilia ya sea escribiendo, leyendo, hablando o escuchando, concluyendo que una de las cuestiones que más inhiben el desempeño exitoso de un grupo es la carencia de una comunicación eficaz, (Ramírez y Hugueth, 2017).
Fundamentación jurídica de la convivencia escolar en Colombia y su vinculación para la construcción de paz

Según la Constitución Política De Colombia en el Artículo 44. Son derechos fundamentales de los niños: la vida, integridad física, salud, seguridad social, alimentación equilibrada, su nombre y nacionalidad, tener una familia y no ser separados de ella, el cuidado, educación, cultura, recreación y libre expresión de su opinión. Serán protegidos contra toda forma de abandono, violencia física o moral, secuestro, venta, abuso sexual, explotación laboral o económica y trabajos riesgosos.

Gozarán también de los demás derechos consagrados en la Constitución, en las leyes y en los tratados internacionales ratificados por Colombia. La familia, la sociedad y el Estado tienen la obligación de asistir y proteger al niño para garantizar su desarrollo armónico e integral y el ejercicio pleno de sus derechos. Cualquier persona puede exigir de la autoridad competente su cumplimiento y la sanción de los infractores, los derechos de los niños prevalecen sobre los derechos de los demás.

Por su parte la Ley General de Educación en su Artículo $13^{\circ}$, presenta los objetivos comunes de todos los niveles. Es objetivo primordial de todos y cada uno de los niveles educativos el desarrollo integral de los educandos mediante acciones estructuradas encaminadas a: formar la personalidad y capacidad de asumir con responsabilidad y autonomía sus derechos y deberes; proporcionar una sólida formación ética-moral, fomentar la práctica del respeto a los derechos humanos; promover en instituciones educativas, prácticas democráticas para el aprendizaje de los principios y valores de la participación 
y organización ciudadana, estimulo de autonomía y responsabilidad.

Al mismo tiempo desarrollar una sana sexualidad que promueva el conocimiento de sí mismo mediante el autoestima, la construcción de la identidad sexual dentro del respeto por la equidad de los sexos, la afectividad, el respeto mutuo y prepararse para una vida familiar armónica y responsable; Crear y fomentar una conciencia de solidaridad internacional; Desarrollar acciones de orientación escolar, profesional y ocupacional; Formar una conciencia educativa para el esfuerzo y el trabajo, y Fomentar el interés y el respecto por la identidad cultural de los grupos étnicos

A su vez, la Ley 1620 de 2013. En el Artículo 1, reglamenta el funcionamiento del Sistema Nacional de Convivencia Escolar y Formación para el ejercicio de los Derechos Humanos, la educación para la sexualidad y la prevención y mitigación de la violencia escolar; sus herramientas; los lineamientos generales bajo los cuales se deben ajustar los manuales de convivencia de los establecimientos educativos, de acuerdo con lo ordenado en la Ley 1620 de 2013 y otros aspectos relacionados con incentivos y la participación de las entidades del orden Nacional y Territorial, establecimientos educativos, la familia y la sociedad dentro del Sistema Nacional de Convivencia Escolar.

El Decreto 1965 de 2013. En el Artículo $1^{\circ}$. Reglamenta el funcionamiento del Sistema Nacional de Convivencia Escolar y Formación para el Ejercicio de los Derechos Humanos, la Educación para la Sexualidad y la Prevención y Mitigación de la Violencia Escolar; sus herramientas; los lineamientos generales bajo los cuales se deben ajustar los Manuales de Convivencia de los Establecimientos Educativos, de acuerdo con lo ordenado en la Ley
1620 de 2013 y otros aspectos relacionados con incentivos y la participación de las entidades del orden nacional y territorial, establecimientos educativos, la familia y la sociedad dentro del Sistema Nacional de Convivencia Escolar.

El artículo $2^{\circ}$, ámbito de aplicación, el decreto se aplicará en todos los establecimientos educativos oficiales y no oficiales de educación preescolar, básica y media del territorio nacional y demás instancias que conforman el Sistema Nacional de Convivencia Escolar y Formación para los Derechos Humanos, Educación para la Sexualidad, y Prevención y Mitigación de la Violencia Escolar, también familiar, social y demás actores que participan en rutas de atención integral.

Finalmente, el decreto 1860 de 1994 en dos artículos que son importantes el 39 , el cual hace referencia al Servicio social estudiantil el cual busca integrar al estudiante con la comunidad, lo cual es fundamental en la formación ciudadana. Vincular por medio de proyectos a la comunidad con la escuela es necesario para el desarrollo de ambas (decreto 1860 DE 1994). El otro es el Artículo 40 que nos informa sobe el Libre desarrollo de la personalidad, el cual se constituye en una herramienta para la orientación del estudiante en la participación y en la relación con los demás. (Decreto 1860 de 1994).

Considerando lo antes en mención, se puede decir que los fundamentos jurídicos de la convivencia escolar en Colombia, están vinculados con la construcción de paz, (ver tabla 2), promoviendo el derecho a la vida, integridad física, salud, seguridad social, alimentación equilibrada, nacionalidad, el cuidado, educación, cultura, recreación y libre expresión de su opinión, a fin de formar la personalidad y la capacidad de asumir con responsabilidad y autonomía los derechos y deberes del niño (a). 
Tabla 2

Fundamento jurídico de la convivencia escolar en Colombia y su vínculo con la construcción de paz

\begin{tabular}{|c|c|c|}
\hline $\begin{array}{c}\text { Fundamento jurídico } \\
\text { de la convivencia } \\
\text { escolar en Colombia }\end{array}$ & $\begin{array}{l}\text { Convivencia escolar y su vínculo } \\
\text { con la construcción de paz }\end{array}$ & $\begin{array}{l}\text { Indicadores de } \\
\text { construcción de paz }\end{array}$ \\
\hline $\begin{array}{l}\text { Constitución Política de } \\
\text { Colombia, artículo } 44 \text {. }\end{array}$ & $\begin{array}{l}\text { Derechos fundamentales del niño (a): la vida, } \\
\text { integridad física, salud, seguridad social, } \\
\text { alimentación equilibrada, nombre y nacionalidad, } \\
\text { tener una familia y no ser separados de ella, el } \\
\text { cuidado, educación, cultura, recreación y libre } \\
\text { expresión de su opinión }\end{array}$ & \multirow{4}{*}{$\begin{array}{l}\text { Comprensión y respeto } \\
\text { de los derechos } \\
\text { humanos. } \\
\text { Concepción } \\
\text { democrática. } \\
\text { Construcción } \\
\text { de relaciones } \\
\text { interpersonales e } \\
\text { institucionales y } \\
\text { culturales justas. } \\
\text { Fortalecimiento de los } \\
\text { valores. }\end{array}$} \\
\hline $\begin{array}{l}\text { Ley General de } \\
\text { Educación en su } \\
\text { artículo } 13^{\circ} .\end{array}$ & $\begin{array}{l}\text { Es objetivo primordial de todos y cada uno de } \\
\text { los niveles educativos el desarrollo integral de } \\
\text { los educandos mediante acciones estructuradas } \\
\text { encaminadas a: Formar la personalidad y la } \\
\text { capacidad de asumir con responsabilidad y } \\
\text { autonomía sus derechos y deberes; Proporcionar } \\
\text { una sólida formación ética-moral, fomentar la } \\
\text { práctica del respeto a los derechos humanos }\end{array}$ & \\
\hline $\begin{array}{l}\text { Ley } 1620 \text { de } 2013, \\
\text { artículo } 1 .\end{array}$ & $\begin{array}{l}\text { Funcionamiento del Sistema Nacional de } \\
\text { Convivencia Escolar y Formación para el ejercicio } \\
\text { de los Derechos Humanos, la educación para la } \\
\text { sexualidad y la prevención y mitigación de la } \\
\text { violencia escolar }\end{array}$ & \\
\hline $\begin{array}{l}\text { Decreto } 1860 \text { de } 1994, \\
\text { artículos } 39 \text { y } 40 .\end{array}$ & $\begin{array}{l}\text { Servicio social estudiantil el cual busca integrar } \\
\text { al estudiante con la comunidad, lo cual es } \\
\text { fundamental en la formación ciudadana. } \\
\text { Libre desarrollo de la personalidad, herramienta } \\
\text { para la orientación del estudiante en la } \\
\text { participación y relación con los demás. }\end{array}$ & \\
\hline
\end{tabular}

Fuente: elaboración propia (2018).

\section{Metodología}

La metodología utilizada en el estudio es de tipo cualitativo con un modelo investigación - acción, orientada a la práctica educativa cuyo propósito es mejorar las mismas. Este modelo implica, la transformación y mejora de una realidad educativa, perspectiva de quien posee el problema, implica colaboración participativa, requiere la reflexión sistemática en el accionar, convierte a la práctica en objeto investigativo, conocer y actuar forma parte del mismo proceso, es realizada por las personas implicadas en la investigación, el elemento de "formación" es esencial en el proceso de investigación.

\section{Participantes}

La población estuvo constituida por cuarenta (40) estudiantes con edades comprendidas entre los 10 y 13 años, del grado sexto de bachillerato de la IED Humberto Velázquez García, del Municipio de Zona Bananera, Magdalena. Cabe descartar, que para efectos de publicación de resultados los participantes diligenciaron debidamente el consentimiento informado.

\section{Técnicas e instrumentos}

La investigación cualitativa podría entenderse como una categoría de diseños de investigación que extraen descripciones 
a partir de observaciones que adoptan la forma de entrevistas, narraciones, notas de campo, grabaciones, transcripciones de audio y vídeo, registros escritos de todo tipo, fotografías o películas y artefactos, (Pérez, 2012).

\section{Procedimiento}

Por su parte, se establece la metodología en siete (7) momentos:

- Momento (1): en conjunto, los docentes investigadores diseñan el plan de acción que incluye la selección de los temas y estrategias investigativas correspondientes; según sus áreas académicas se seleccionan las temáticas, respondiendo a tres criterios: (a) áreas de humanidades, temas extensos y donde los estudiantes han demostrado bajo desconocimiento $\mathrm{y}$ (b) áreas de convivencia, temas de interés para los estudiantes. Asimismo, se diseña un registro anecdótico como diagnóstico y evaluación.

- Momento (2): los docentes a través de la diagnosis, diseñan según el modelo de las pruebas estandarizadas que identifican indicadores para generar espacios de construcción de paz y convivencia, con análisis descriptivo.

- Momento (3): se despliega la implementación y transversalización de las estrategias investigativas dirigidas a los estudiantes y orientadas por los docentes.

- Momento (4): desde el área de tecnología e informática se desarrolla la formación en la creación estrategias para generar espacios de construcción de paz y convivencia a través de la investigación como estrategia pedagógica (IEP), apropiación desde la praxis basándose en temas de in- terés, y posteriormente elaborados y consolidados como resultado de la indagación de los temas señalados por las áreas específicas.

- Momento (5): Aplicación del instrumento de evaluación, diseñado según el modelo de las pruebas estandarizadas que identifican los elementos de espacios de construcción de paz y convivencia a través de la investigación como estrategia pedagógica (IEP), análisis descriptivo

- Momento (6): Análisis e interpretación de resultados.

- Momento (7): Reflexión sobre la generación de espacios de construcción de paz y convivencia a través de la investigación como estrategia pedagógica (IEP).

\section{Resultados}

A continuación, se ofrece una síntesis de los resultados obtenidos después de revisar las pruebas estandarizadas que identifican indicadores para generar espacios de construcción de paz y convivencia, el análisis descriptivo de cada una de las situaciones vinculadas a la comunidad en referencia al comportamiento de los estudiantes, se pudo valorar comportamientos inadecuados en clases, destrucción de objetos, uso inadecuados de los dispositivos móvil, falta de respeto a los compañeros, evasión de clases, peleas y agresiones, en tal sentido, convienen replantear con estrategias basadas en valores para mejorar la sana convivencia a través de la IEP apoyadas en las TIC, esto con la finalidad en disminuir los problemas convivenciales que afectan a la comunidad objeto de esta investigación, el indicador menos presente fue la comprensión y respeto de los derechos humanos. 
Tabla 3

Indicadores para promover la construcción de pazy convivencia escolar a través desde la IEP apoyada en TIC

\begin{tabular}{lll}
\hline \multicolumn{1}{c}{ Variable } & \multicolumn{1}{c}{ Indicadores } & \multicolumn{1}{c}{ La IEP apoyada en TIC } \\
\hline & $\begin{array}{l}\text { Reconocimiento, comprensión y respeto de los } \\
\text { derechos humanos. }\end{array}$ & $\begin{array}{l}\text { Identificar la manera como se ha } \\
\text { constituido la educación. }\end{array}$ \\
$\begin{array}{l}\text { Construcción de } \\
\text { paz y convivencia }\end{array}$ & $\begin{array}{l}\text { Concepción democrática, construcción de } \\
\text { escolaciones interpersonales e institucionales y }\end{array}$ & $\begin{array}{l}\text { Como se conformó el pensamiento } \\
\text { pedagógico. }\end{array}$ \\
& $\begin{array}{l}\text { Fulturales justas. } \\
\text { Fundamenta una de esas líneas } \\
\text { metodológicas. }\end{array}$ \\
\hline
\end{tabular}

Fuente: elaboración propia (2018).

La IEP apoyada en TIC, busca la reflexión/sistematización e investigación donde los participantes mediante la negociación y diálogo de saberes, analizan su práctica de acompañamiento, coinvestigación e investigación e identifican aciertos, desaciertos y fortalezas, para configurar nuevas mediaciones pedagógicas; donde el conocimiento, la tecnología e innovación predominan y hacen necesario que las culturas infantiles y juveniles se preparen para moverse en un mundo que ha sido conformado desde estas nuevas realidades. Por tal motivo, se pueden establecer indicadores para promover la construcción de paz y convivencia escolar a través desde la IEP apoyada en TIC, (ver tabla 3).

El registro anecdótico como diagnóstico y evaluación se realizaron a docentes y estudiantes para que estos manifiesten su inquietud acerca de los métodos de enseñanza de la institución y de la convivencia de la misma, y si están consciente de implementar estrategia pedagógica para que las clases sean primero motivantes y luego dejen un aprendizaje que los lleve a valorarse y a valorar al otro. $\mathrm{Al}$ mismo tiempo se pudo vivenciar la personalidad y capacidad de asumir con responsabilidad y autonomía los derechos y deberes de los niños(as) y como esto impacta en su comportamiento.
Para generar espacios de construcción de paz y convivencia a través de la investigación como estrategia pedagógica (IEP), se diseñaron estrategias de formación para propiciar cambios de paradigmas mentales y conductuales, involucrando familia, escuela y sociedad, a partir de los hábitos, creencias, actitudes que incluyen los valores. Por tal razón la integración de la IEP y las TIC para la construcción de paz y convivencia al aula de clase se convierte en una estrategia pedagógica que permite generar espacios de reflexión y discusión que propicien una formación en valores y competencias ciudadanas, con un enfoque dirigido al desarrollo de habilidades para la convivencia y la paz, participación y responsabilidad democrática y la pluralidad, identidad y valoración de las diferencias, que busque la inclusión y dignidad social de la escuela.

A través de esta observación directa se pudo determinar cuál es el verdadero interés de ese grupo estudiantil, sus necesidades tanto psíquicas, físicas, afectivas y sobre todo de índole convivencial. Los resultados presentan una tendencia favorable frente a las charlas que les brindan los docentes a sus estudiantes y la forma que les dan las clases. A través de este registro de observación se pudo verificar la forma como desarrollan las 
clases los docentes, que dinámica utiliza, cuál es su trato con los estudiantes, se observó por un periodo las clases de algunos docentes, los estudiantes y docentes en horas de descanso, en actividades que desarrolla la institución, verificándose la actitud frente a las clases por lo general fueron observaciones sin ninguna prevención a los observados, donde se hizo presente la falta de valores hacia el otro, como una sólida formación ética-moral, los que va en detrimento con la práctica del respeto a los derechos humanos.

\section{Conclusiones}

La paz y la convivencia escolar a través de la IEP apoyada en TIC constituyen hoy en día un medio eficaz y dinámico dentro de cualquier institución, organismo, empresas y en general, donde el hombre y la mujer, es capaz de realizar actividades dentro de la sociedad en la que se desenvuelve. Dadas las condiciones que anteceden, la cual consiste en la implementación y puesta en marcha de actividades curriculares, basadas en una serie de estrategias pedagógicas, fundadas en valores, esto con los propósitos ya expresados con referencia al mejoramiento de la convivencia entre los estudiantes, del grado sexto de la IED Humberto Velásquez García, sede Palomar.

$\mathrm{Al}$ generar espacios de construcción de paz y convivencia a través de la investigación como estrategia pedagógica (IEP), se evidencia en la población objeto de estudio, poca comprensión y respeto de los derechos humanos, falta de concepción democrática, lo que desmejora la construcción de relaciones interpersonales e institucionales y culturales justas, propiciando falencias en el fortalecimiento de los valores en las instituciones.
Por lo tanto, se cree que la evaluación de tales propósitos tiene que estar asociada al desarrollo de la malla curricular, al desempeño actitudinal de los estudiantes, $\mathrm{y}$, sobre todo, que es lo más importante a la generación de un buen clima escolar en donde se evidencie la tolerancia, cooperación, aceptación de las diferencias y crecimiento del ambiente de alegría, autoestima, respeto y solidaridad entre todos.

En tal sentido se plantea evaluar a través de la observancia de los resultados comportamentales de los estudiantes desde todas las áreas, así como por medio de la revisión de las actas de compromiso disciplinarias, donde se registran los casos de indisciplina que se propician por actitudes de intolerancia e irrespeto. Como constatación de la generación del clima institucional y el afianzamiento de la propuesta pedagógica a través de la IEP apoyadas en las TIC.

Esta propuesta no es un trabajo aun terminado, sino que, por el contrario, pretende ser un punto de partida, referente $u$ ejemplo que permita la vinculación de todas las áreas del conocimiento visibilizando la propuesta en valores que exige la Ley de Educación Nacional, y que otras instituciones retomen esta iniciativa adaptada a sus intereses, necesidades y contexto, donde la acción reflexiva sobre el que hacer educativo sea una condición permanente, fomentando así la opción de que se enriquezca el proceso de convivencia y de aprendizaje $y$ en especial en los educandos que se formen con el desarrollo de competencias tanto para la vida, como para las nuevas exigencias socio-laborales que plantean las dinámicas globales y, por ultimo; que el que hacer educativo potencie el saber hacer, el saber y el ser, dentro del proceso de enseñanza - aprendizaje. 


\section{Referencias}

Belloch. (2012). Las Tecnologías de la Información y Comunicación en el aprendizaje. Universidad de Valencia.

Carbajal, P. (2013). Convivencia democrática en las escuelas. Apuntes para una reconceptualizacion. Revista Iberoamericana de Evaluación Educativa, 6 (2), 13-35.

Cárdenas, N., \& Angulo, F. (2016). Análisis de las dimensiones de adaptación, mejoramiento e innovación en los procesos de aprendizaje tecnológico. Cultura Educación y Sociedad, 7(2), 139-149.

Cerdas, E. (2015). Desafíos de la educación para la paz hacia la construcción de una cultura de paz. Revista Electrónica Educare, 19(2).

Fernández, O. (2006). Una aproximación a la cultura de paz en la escuela. Revista Educere V.10 N.33.

Herrera, B. (2013). La constitucionalización de los derechos del consumidor en Colombia: un análisis desde los derechos sociales fundamentales. Civilizar. Ciencias Sociales y Humanas, 13 (25), 33-47.

Mejía, M. (2011). La investigación como estrategia pedagógica una apuesta por construir pedagogías críticas en el siglo XXI. Praxis \& saber. Vol. 2. Núm. 4.

Mineducación. (2016). Eduderechos. Recuperado el 15 de octubre de 2017, de Ministerio de Educación Nacional: http://www.mineducacion.gov. co/1759/ w3-propertyvalue-55309.html

MinEducación. (2016). Propuesta de desempeños de educación para la Paz, para ser enriquecidas por los docentes de Colombia. Recuperado el 15 de octubre de 2017, de Ministerio de Educación Nacional: http://www. mineducacion. gov.co/

MinEducación. (2016). Secuencias didácticas de educación para la Paz, para ser enriquecidas por los docentes de Colombia. Recuperado el 15 de octubre de 2016, de Ministerio de Educación Nacional: http://www.mineducacion. gov.co/

Ministerio de Educación Nacional. Ley 115 de 1994. Ley General de la Educación, 1994.

Ministerio de Educación Nacional. Ley 70. Decreto 804 de 1995. Decreto 1290. Decreto 804 del 1995.

Ministerio de Educación Nacional. Decreto 1965 de 2013. Min-educación. Recuperado de: https://www.mineducacion. gov.co/1621/articles-328630_archivo_ pdf_Decreto_1965.pdf.

Ministerio de Educación Nacional. Ley 1620 de 2013. Min-Educación. Recuperado de: https://www.mineducacion. gov.co/1759/articles327397_archivo_ pdf_proyecto_decreto.pdf.

Ministerio de Educación Nacional. Decreto 1860 de agosto 3 de 1994. MinEducación. Recuperado de: https:// www.mineducacion.gov.co/1621/articles-86240_archivo_pdf.pdf

Presidencia de la República de Colombia. 1991. Constitución política de Colombia. Recuperado de: http://wsp.presidencia.gov.co/Normativa/Documents/ Constitucion-Politica-Colombia. Pdf

Pick, S. (2002). Formación cívica y ética/ Civics and Ethics. Editorial Limusa.

Ramírez Molina Reynier Israel y Hugueth Alfredo. (2017). Modelo de comunicación productiva para las organizaciones de salud pública en Venezuela. Revista Opción. Año 33, N 83. Pág.: 305-335. Universidad del Zulia. Maracaibo (Venezuela).

Robles, A. (2015). Propuesta de convivencia escolar a través de talleres de prevención de violencia escolar con perspectiva de género. Cultura Educación y Sociedad, 6(2).

Samper, J., \& Maussa, E. (2014). Desarrollo moral y competencias ciudadanas en la juventud universitaria. Jurídicas CUC, 10(1), 43-60. 